\title{
Pequenos mamíferos não-voadores da Reserva Florestal do Morro Grande - distribuição das espécies e da diversidade em uma área de Mata Atlântica
}

\author{
Renata Pardini ${ }^{1}$ \& Fabiana Umetsu ${ }^{2}$ \\ Biota Neotropica v6 (n2)-http://www.biotaneotropica.org.br/v6n2/pt/abstract?article+bn00606022006 \\ Recebido em 17/11/2004 \\ Versão reformulada recebida em 21/3/2005 \\ Publicado em 01/05/2006

\begin{abstract}
${ }^{1}$ Departamento de Zoologia, Instituto de Biociências, Universidade de São Paulo, Rua do Matão, 321, travessa 14, 05508-900 São Paulo, SP (autor para correspondência: renatapardini@uol.com.br)

${ }^{2}$ Departamento de Ecologia, Instituto de Biociências, Universidade de São Paulo, Rua do Matão, 321, travessa 14,
\end{abstract} \\ 05508-900 São Paulo, SP
}

\begin{abstract}
Pardini, R. and Umetsu, F. Non-volant small mammals from the Morro Grande Forest Reserve - distribution of species and diversity in an Atlantic Forest area. Biota Neotrop. May/Aug 2006 vol. 6 no. 2, http://www.biotaneotropica.org.br/ v6n2/pt/abstract?article+bn00606022006. ISSN 1676-0603.
\end{abstract}

Non-volant small mammals, the most diverse ecological group of mammals in Neotropical forests, play an important role in forest dynamics and are good indicators of both local and landscape alterations. However, little is known about species and diversity distribution and only a few of the largest Atlantic Forest remnants have been adequately sampled. Based on a survey we carried out in the Morro Grande Forest Reserve, São Paulo State, and on other 20 Atlantic forest inventories, this study aims at (1) describing the non-volant small mammal list and community structure of the Reserve, (2) describing how species and diversity are distributed in space and time in the Reserve and (3) investigating how diversity is affected by capture methods. The non-volant small mammal fauna of the Reserve includes several rare and mature forest species, besides common species from genera that are usually abundant in other well preserved forests. The total number of species is high, in part due to the use of large pitfall traps in the sampling protocol, but also probably due to the Reserve location and altitude. The additive partitioning of diversity indicates that a major part of diversity is found locally in sample sites, a second part among sample sites within the same habitat type and just a minor part among habitats, suggesting the importance of micro-scale forest heterogeneity to the distribution of non-volant small mammals. Abundance and richness did not vary between the two sampled years and it is possible that continuous forest areas may present more temporally stable populations and communities. However, they varied seasonally, with high values found at the end of the wet season and low values at the end of the dry season. Pitfall traps showed to be extremely efficient for capturing non-volant small mammals.

Key words: Atlantic forest, inventories, non-volant small mammals, diversity patterns, sampling methods, additive partitioning, inter-annual variations, seasonal variations, spatial variations, habitat fragmentation, pitfall traps 


\section{Resumo}

Pardini, R. and Umetsu, F. Pequenos mamíferos não-voadores da Reserva Florestal do Morro Grande - distribuição das espécies e da diversidade em uma área de Mata Atlântica. Biota Neotrop. May/Aug 2006 vol. 6 no. 2, http:// www.biotaneotropica.org.br/v6n2/pt/abstract?article+bn00606022006. ISSN 1676-0603

Pequenos mamíferos não-voadores, grupo ecológico mais diversificado de mamíferos das florestas Neotropicais, influenciam a dinâmica florestal e são bons indicadores de alterações locais do habitat e da paisagem. Entretanto, padrões de distribuição das espécies e da diversidade são pouco conhecidos e poucos dos maiores remanescentes de Mata Atlântica foram inventariados adequadamente. Utilizando o levantamento por nós realizado na Reserva Florestal do Morro Grande, São Paulo, e outros 20 levantamentos de pequenos mamíferos não-voadores realizados em áreas de Mata Atlântica, este trabalho tem por objetivo (1) apresentar a lista de espécies e a estrutura da comunidade de pequenos mamíferos nãovoadores da Reserva, (2) descrever como a diversidade se distribui no espaço e no tempo na Reserva e (3) investigar como a diversidade destes animais é afetada pelos métodos de amostragem. A fauna de pequenos mamíferos não-voadores da Reserva inclui muitas espécies raras e características de matas maduras, e espécies comuns pertencentes a gêneros que em geral dominam outras áreas grandes e bem preservadas de Mata Atlântica. O número total de espécies é elevado, em parte devido à inclusão de armadilhas de queda na amostragem, mas provavelmente também por sua localização e altitude. A partição aditiva da diversidade indica que a maior parte da diversidade é encontrada localmente nos sítios de amostragem, secundariamente entre sítios do mesmo habitat e apenas uma menor parte entre habitats, indicando a importância da heterogeneidade da floresta em micro-escala para a distribuição de pequenos mamíferos não-voadores. A abundância e a riqueza não variaram entre os dois anos de amostragem, sendo possível que as populações e comunidades de matas contínuas sejam mais estáveis temporalmente. Porém, variaram sazonalmente, com valores altos no final da estação chuvosa e baixos no final da estação seca. As armadilhas de queda mostraram-se extremamente eficientes na captura de pequenos mamíferos não-voadores.

Palavras-chave: Mata Atlântica, inventário, pequenos mamíferos não-voadores, padrões de diversidade, métodos de amostragem, partição aditiva da diversidade, variações interanuais, variações sazonais, variações espaciais, fragmentação de habitat, armadilhas de queda 


\section{Introdução}

Marsupiais e pequenos roedores formam o grupo ecológico mais diversificado de mamíferos das florestas Neotropicais, com mais de 190 espécies atualmente reconhecidas para o Brasil e cerca de 92 espécies na Mata Atlântica, das quais quase metade (43 espécies) é endêmica deste bioma (Fonseca et al. 1996). Estes números devem ser ainda mais altos, e várias espécies foram descritas nos últimos anos (Patterson 2000, Grelle 2002). Além disso, ainda existem grandes lacunas no conhecimento atual da distribuição geográfica das espécies e da biogeografia desses grupos na Mata Atlântica. Estudos filogeográficos para marsupiais e roedores indicam que a Mata Atlântica é ocupada por dois componentes faunísticos historicamente distintos que residem em duas áreas biogeográficas bem definidas, uma ao norte e outra ao sul (Costa 2003). Estes dois componentes, ambos com várias espécies endêmicas (Costa et al. 2000), parecem estar mais relacionados com outras áreas Neotropicais, como a Amazônia ou o Brasil Central, do que entre si (Costa 2003). Com base na distribuição dos mamíferos endêmicos, Vivo (1997) reconhece quatro sub-regiões mastofaunísticas para a Mata Atlântica, que subdividem o componente norte descrito acima nas regiões Nordeste (ao norte do rio São Francisco) e Sudeste da Bahia, e o componente sul, nas regiões do Rio de Janeiro e Sul (ao sul do Trópico de Capricórnio). A fauna de pequenos mamíferos não-voadores da Mata Atlântica no Estado de São Paulo, embora com poucas espécies endêmicas, abrange espécies de sub-regiões distintas (sensu Vivo 1997), de distribuição temperada e tropical.

Além de sua importância numérica, o conjunto das informações sobre a ecologia das espécies e das comunidades de pequenos mamíferos não-voadores indica que marsupiais e pequenos roedores exercem influência importante na dinâmica das florestas Neotropicais e são bons indicadores tanto de alterações locais do habitat como alterações da paisagem. A influência na dinâmica da floresta se dá, principalmente, através da predação do banco de sementes e de plântulas (Pizo 1997, Sánchez-Cordero \& Martinez-Gallardo 1998, Vieira et al. 2003a) e da dispersão de sementes e fungos micorrízicos (Janos et al. 1995, Brewer \& Rejmanek 1999, Grelle \& Garcia 1999, Vieira \& Izar 1999, Mangan \& Adler 2000, Colgan \& Claridge 2002, Pimentel \& Tabarelli 2004). Já as relações estreitas com alterações locais do habitat devem estar associadas à especificidade, entre os pequenos mamíferos não-voadores, do uso de microhabitats. Aparentemente, a partição de recursos e a coexistência das espécies são facilitadas pela ocupação diferencial dos estratos da floresta (Leite et al. 1997, Cunha \& Vieira 2002, Grelle 2003, Vieira \& Monteiro-Filho 2003). Como conseqüiência, a complexidade da vegetação parece estar relacionada positivamente ao número de espécies (Grelle 2003) ou à abundância total de pequenos mamíferos não-voadores em áreas de Mata Atlântica (Gentile \&
Fernandez 1999, Pardini et al. 2005). Nas florestas mais jovens ou mais alteradas, onde o dossel é mais aberto e o subbosque mais denso, diminuem ou desaparecem as espécies florestais que usam preferencialmente o dossel (Malcolm 1995) ou são exclusivamente terrestres (Vieira et al. 2003b, Pardini 2004, Pardini et al 2005), e proliferam aquelas espécies florestais que usam preferencialmente o sub-bosque (Vieira et al. 2003b, Pardini 2004, Pardini et al. 2005). Além disso, a maioria das espécies da Mata Atlântica não é capaz de ocupar áreas abertas, como pastagens ou campos artificiais, os quais são dominados por espécies generalistas e características de formações abertas como o Cerrado (Stallings 1989, Stevens \& Husband 1998, Feliciano et al. 2002) ou, no caso dos campos naturais de altitude, por espécies restritas a este habitat (Bonvicino et al. 1997). Como conseqüência, as taxas de dispersão de indivíduos entre fragmentos de Mata Atlântica isolados e inseridos em uma matriz de ambientes abertos são bastante baixas (Pires et al. 2002), levando a extinções locais (Castro \& Fernandez 2004). Assim, a diversidade se modifica em paisagens com baixa proporção de florestas nativas, onde fragmentos de florestas pequenos e isolados apresentam menor riqueza, mas maior variabilidade na composição de pequenos mamíferos nãovoadores, do que fragmentos maiores ou conectados por corredores (Pardini et al. 2005).

Os padrões de distribuição das espécies, de distribuição da diversidade e de estrutura das comunidades de pequenos mamíferos não-voadores relacionados aos amplos gradientes ambientais observados no bioma Mata Atlântica ainda são pouco conhecidos. As comunidades diferem entre florestas de baixada e de altitude (Bonvicino et al. 1997, Vieira 1999, Vivo \& Gregorin 2001, Vieira \& Monteiro-Filho 2003, Geise et al. 2004) e entre florestas em diferentes estádios de regeneração ou níveis de perturbação (Vieira 1999, Pardini 2004, Pardini et al. no prelo). Existem poucas informações sobre como a diversidade de uma região de mata contínua varia ao longo do tempo, é afetada pelas técnicas de amostragem, ou está relacionada à heterogeneidade ambiental em micro e meso escalas. Apesar da enorme ameaça que a Mata Atlântica sofreu e ainda vem sofrendo e da drástica redução da sua área de cobertura, a maioria dos grandes remanescentes deste bioma ainda não foi inventariada adequadamente. Com poucas exceções (Reserva Biológica de Una, BA - Pardini 2004; Parque Estadual do Rio Doce, MG - Stallings 1989, Grelle 2003; Parque Estadual Intervales, SP-Olmos 1991, Vivo \& Gregorin 2001, Vieira \& Monteiro-Filho 2003; Parque Nacional do Itatiaia - Geise et al. 2004; Parque Nacional do Caparaó Bonvicino et al. 1997), os levantamentos de pequenos mamíferos não-voadores em áreas contínuas de Mata Atlântica são pontuais no espaço e, muitas vezes, também no tempo. Conhecimento sobre a variação temporal e espacial das espécies e da diversidade em Unidades de Conservação ou outras áreas contínuas é fundamental para orientar planos 
de manejo e conservação, mas também para o estabelecimento de parâmetros para comparação com áreas degradadas ou fragmentadas, ou seja, para o entendimento de como os padrões temporais e espaciais da distribuição das espécies e da diversidade são afetados pelas alterações antrópicas em micro e meso escalas.

Neste trabalho, temos como objetivo (1) apresentar e comentar a lista de espécies e a estrutura da comunidade de pequenos mamíferos não-voadores de uma área de Mata Atlântica contínua (Reserva Florestal do Morro Grande, SP), (2) descrever como a diversidade se distribui no espaço e no tempo na Reserva e (3) investigar como a diversidade destes animais é afetada pelos métodos de amostragem utilizados.

\section{Material e Métodos}

\section{1. Área e sítios de estudo}

Amostramos seis sítios na Reserva Florestal do Morro Grande, três em floresta secundária (A, B e C) e três em floresta madura (Grilos, Quilombo e Torres). As características da Reserva como tipo de vegetação, relevo, clima, coordenadas geográficas e histórico de perturbação podem ser encontrados em Metzger e colaboradores (2006).

\section{Coleta de dados}

Os levantamentos de pequenos mamíferos nãovoadores foram realizados ao longo de dois anos e quatro meses utilizando dois tipos de armadilhas.

\subsection{Armadilhas de queda}

Em cada um dos seis sítios, foram instalados 11 baldes de $60 \mathrm{l}$, um a cada $10 \mathrm{~m}$, conectados por cercas-guia (com 50 cm de altura), resultando em uma série em linha de 100 m de extensão. Foram realizadas quatro sessões de captura de oito dias cada, duas entre janeiro e fevereiro de 2002, e duas um ano depois, entre dezembro de 2002 e janeiro de 2003, em que todos os seis sítios foram amostrados simultaneamente. No total, foram realizados 32 dias de amostragem com um esforço de 352 armadilhas-noite para cada sítio e 2.112 armadilhas-noite na Reserva Florestal do Morro Grande.

\subsection{Armadilhas Sherman}

Em cada um dos seis sítios, foram estabelecidas duas linhas paralelas de $165 \mathrm{~m}$, distantes $20 \mathrm{~m}$ uma da outra. Cada linha continha 12 estações separadas por 15 m de distância, totalizando 24 estações por sítio. Uma das linhas se sobrepunha à linha de armadilhas de queda. Em cada estação, foram dispostas duas armadilhas Sherman, uma maior (37,5 x 10 x $12 \mathrm{~cm}$ ) e uma menor ( 23 x 7,5 x 8,5 cm), totalizando 48 armadilhas por sítio. As armadilhas em cada estação foram colocadas a duas alturas, no chão e a cerca de dois metros de altura no sub-bosque, e a altura das armadilhas maiores e menores foi alternada entre estações adjacentes. No total, foram realizadas três sessões de captura de sete dias cada, uma em julho de 2003, outra em outubro de 2003 e a última em abril de 2004, totalizando 21 dias de captura, 1.008 armadilhasnoite por sítio e 6.048 armadilhas-noite na Reserva Florestal do Morro Grande. Para cada sessão de captura, três sítios foram amostrados ao mesmo tempo e o total dos seis sítios foram amostrados dentro de um período de um mês.

Parte dos indivíduos capturados foi coletada para determinação do número cromossômico, para a identificação das espécies e para a formação de uma coleção de referência. Os demais foram marcados com brincos metálicos numerados (Fish and small animal tag-size 1- National Band and Tag Co., Newport, Kentucky) e posteriormente soltos. Os espécimes coletados foram depositados no Museu de Zoologia da Universidade de São Paulo. Já que a área amostrada e o protocolo de captura foram os mesmos para todos os sítios, utilizamos o número de indivíduos capturados por espécie como um índice da abundância relativa (Slade \& Blair 2000).

\section{Análise de dados}

\subsection{Lista de espécies}

Para comparar a riqueza, a composição e a estrutura da comunidade de pequenos mamíferos não-voadores da Reserva Florestal do Morro Grande, utilizamos outros 20 levantamentos realizados em áreas de Mata Atlântica com mais de 700 ha (Apêndice 1).

\subsection{Distribuição espacial da diversidade e estimativas de riqueza}

Utilizamos dois métodos para descrever como a diversidade de pequenos mamíferos não-voadores está distribuída na Reserva Florestal do Morro Grande. A partir da partição aditiva da diversidade total encontrada na Reserva (diversidade $\gamma$ ) em dois componentes, diversidade alfa e diversidade beta $(\gamma=\alpha+\beta)$, calculamos o número de espécies que se encontrou em média por sítio de amostragem (diversidade $\alpha$ ) e o número de espécies que em média não foi encontrado em um sítio de amostragem único (diversidade $\beta$ ) (Lande 1996, Veech et al. 2002). Uma das vantagens da partição aditiva da diversidade em relação ao conceito tradicional multiplicativo de Whittaker (1960) é que os dois componentes, diversidade dentro de sítios $(\alpha)$ e diversidade entre sítios ( $\beta$ ), têm a mesma unidade e podem ser vistos como proporções da diversidade total $(\gamma)$, permitindo inferências sobre os mecanismos biológicos associados à diversidade (Veech et al. 2002). A partição aditiva também possibilita a inclusão de múltiplas escalas espaciais, sendo que a diversidade $\gamma$ em uma escala, passa 
ser a diversidade á na escala seguinte (Veech et al. 2002). Neste trabalho, calculamos a diversidade á considerando os seis sítios de amostragem na Reserva Florestal do Morro Grande individualmente ( $\alpha 1$ = média do número de espécies por sítio), a diversidade á por habitat ( $\alpha 2$ = média do número de espécies por tipo de floresta, secundária ou madura), a diversidade ã para a Reserva ( $\gamma$ = número total de espécies considerando todos os seis sítios), a diversidade $\beta$ entre sítios do mesmo habitat $(\beta 1=\alpha 2-\alpha 1)$ e a diversidade $\beta$ entre habitats ( $\beta 2=\gamma-\alpha 2)$. Posteriormente, calculamos a proporção da diversidade $\gamma$ que corresponde aos componentes $\alpha 1, \beta 1$ e $\beta 2(\gamma=\alpha 1+\beta 1+\beta 2)$. Complementarmente, construímos uma curva média de acúmulo de espécies com o aumento do número de réplicas espaciais e estimamos o número de espécies para a Reserva Florestal do Morro Grande utilizando o estimador nãoparamétrico Jacknife 2 no programa EstimatesS (Cowell 2000). Tanto a partição aditiva quanto a curva média de acúmulo de espécies foram realizadas também para a Reserva Biológica de Una, que representa o único entre os 20 inventários publicados (Apêndice 1) que utilizou forma de amostragem equivalente à utilizada neste trabalho para a Reserva Florestal do Morro Grande. Oito sítios distribuídos em dois habitats (interior de remanescente de mata madura e borda de remanescente de mata madura) foram amostrados na Reserva Biológica de Una, também utilizando armadilhas Sherman e de queda, ao longo de período de tempo semelhante (Pardini 2004).

\subsection{Distribuição temporal da diversidade e abundância}

Uma vez que a coleta de dados com cada um dos métodos de captura não ocorreu simultaneamente, utilizamos os resultados separados para cada um dos métodos. Comparamos o número de espécies e o número de indivíduos (no total e por espécie) obtidos em cada sítio entre diferentes sessões de captura através de testes $t$ pareados, no caso das duas sessões com intervalo de um ano com armadilhas de queda, e ANOVAs de medidas repetidas, no caso das três sessões de captura dentro do período de um ano com armadilhas Sherman. Neste último caso, só pudemos analisar a variação de abundância de seis das 14 espécies, pois as demais não foram capturadas em uma ou duas das três sessões de captura com armadilhas Sherman, inviabilizando a realização da ANOVA de medidas repetidas.

\subsection{Distribuição da diversidade e abundância entre métodos de amostragem}

Para verificar como as estimativas de diversidade e abundância das espécies de pequenos mamíferos nãovoadores são influenciadas pelo método de captura, realizamos testes $t$ pareados comparando o número de espécies e o número de indivíduos (no total e por espécie) obtidos em cada sítio entre os dois métodos.

\section{Resultados}

\section{Lista de espécies de pequenos mamíferos não- voadores da Reserva Florestal do Morro Grande}

No total, capturamos 592 indivíduos pertencentes a 23 espécies de pequenos mamíferos não-voadores na Reserva Florestal do Morro Grande, sendo oito marsupiais e 15 roedores (Tabela 1). O número de espécies variou de 11 a 18 por sítio com valores mais altos para os sítios de floresta madura (Tabela 1).

\section{Distribuição espacial da diversidade e estimativas de riqueza}

As curvas médias de acúmulo de espécies com aumento de sítios amostrados estão ilustradas na Figura 1. A Reserva Florestal do Morro Grande apresenta um maior número de espécies de pequenos mamíferos não-voadores do que a Reserva Biológica de Una, tanto no total como para qualquer número de sítios, indicando maior diversidade alfa. Além disso, a curva para a Reserva Florestal do Morro Grande é mais inclinada, indicando maior diversidade beta, ou seja, maior heterogeneidade de composição entre sítios, do que a Reserva Biológica de Una. Com seis sítios amostrados, 23 espécies foram observadas e 29,4 estimadas para a Reserva Florestal do Morro Grande. Para a Reserva Biológica de Una, com oito sítios amostrados, foram observadas 14 espécies e estimadas 17,6.

A partição aditiva da diversidade para as duas reservas é mostrada, em termos absolutos, na Tabela 2 e, em termos proporcionais à diversidade total, na Figura 2. Em todas as escalas espaciais, a diversidade local ( $\alpha 1, \alpha 2$ e $\gamma)$ e a diversidade entre locais $(\beta 1, \beta 2)$ são maiores para a Reserva Florestal do Morro Grande do que para a Reserva Biológica de Una (Tabela 2). Entretanto, a distribuição proporcional é bastante semelhante entre as duas reservas (Figura 2), com a maior parte da diversidade (mais de $50 \%$ nos dois casos) sendo encontrada localmente nos sítios de amostragem. A segunda maior proporção da diversidade se deve a diferenças entre sítios do mesmo habitat e, por último, a diferenças entre habitats (Figura 2).

\section{Distribuição temporal da diversidade e abundância}

A abundância das espécies de pequenos mamíferos não-voadores, a abundância total e a riqueza da comunidade não variaram significativamente entre os dois anos amostrados com armadilhas de queda (Tabela 3). Porém, houve variações sazonais significativas ao longo do ano entre as três sessões de captura com armadilhas Sherman (Tabela 4). A abundância total e a riqueza da comunidade foram menores no final da estação seca (outubro) e maiores no final da estação chuvosa (abril). Apesar de marginalmente 
significativo para apenas uma espécie (O. russatus), três das seis espécies analisadas apresentam abundância média maior em abril e quatro apresentam abundância média menor em outubro (Tabela 4).

\section{Distribuição da diversidade e abundância en- tre métodos de amostragem}

O número de indivíduos e o número de espécies capturados em armadilhas Sherman foram significativamente menores do que os capturados em armadilhas de queda (Tabela 5). Nenhuma espécie foi capturada exclusivamente em armadilhas Sherman e das 13 espécies capturadas com os dois métodos apenas uma foi significativamente mais capturada com estas armadilhas (Oryzomys russatus Tabela 5). Por outro lado, 10 espécies foram capturadas exclusivamente em armadilhas de queda. Além destas, duas espécies ( $M$. incanus e $O$. nigripes) foram significativamente mais capturadas, e três ( $D$. aurita, $T$. nigrita e $J$. pictipes) tenderam a ser mais capturadas em armadilhas de queda (Tabela 5).

\section{Discussão}

\section{Composição e riqueza de pequenos mamíferos não-voadores da Reserva Florestal do Morro Grande}

A fauna da Reserva inclui 11 espécies endêmicas do bioma Mata Atlântica (Tabela 1). Além disso, uma espécie é considerada ameaçada de extinção (Rhagomys rufescens) pela Nova Lista da Fauna Brasileira Ameaçada de Extinção do IBAMA e duas são insuficientemente conhecidas para que sejam inseridas em categorias de ameaça (Monodelphis scalops e M. americana), segundo o Workshop da Fundação Biodiversitas que fundamentou a lista do IBAMA (Tabela 1). Rhagomys rufescens é considerado um dos roedores mais raros da Mata Atlântica, do qual não se obteve registro ou coleta durante várias décadas até recentemente, quando a espécie foi re-descrita com base em dois exemplares coletados em São Paulo e Minas Gerais (Percequillo et al. 2004). Os marsupiais terrestres do gênero Monodelphis, por outro lado, são mais comuns, porém são raramente capturados em levantamentos que não utilizam armadilhas de queda, o que explica a falta de informação sobre as espécies deste gênero na literatura. Dos 17 indivíduos capturados na Reserva Florestal do Morro Grande, 16 foram capturados em armadilhas de queda.

Uma outra espécie relativamente rara encontrada na Reserva é Blarinomys breviceps, um roedor endêmico da Mata Atlântica de hábitos semi-fossoriais, também raramente capturado em armadilhas convencionais e com poucos registros de ocorrência confirmada no bioma (Silva et al. 2003). A fauna da Reserva inclui ainda uma espécie rara (um único indivíduo capturado) ainda não descrita, e que aparentemente pertence a um gênero não descrito da tribo Oryzomyini (A. Percequillo, comunicação pessoal).

Além das espécies naturalmente raras, a Reserva Florestal do Morro Grande abriga espécies que aparentemente estão mais associadas a matas maduras. Nestas matas, capturamos 22 espécies, enquanto nas matas secundárias, apesar do maior número de indivíduos capturados, apenas 16 espécies foram encontradas. Estágios mais iniciais de regeneração apresentam em geral maior produtividade e concentram maior proporção de biomassa nas folhas do que em madeira (Guariguata \& Ostertag 2001), provavelmente oferecendo maior disponibilidade de frutos e artrópodes, principais itens alimentares dos pequenos mamíferos não-voadores em florestas Neotropicais. De fato, a densidade de folhagem é positivamente correlacionada à biomassa de artrópodes (Malcolm 1997a) e a disponibilidade de frutos carnosos no sub-bosque é maior em matas em estágios sucessionais iniciais (DeWalt et al. 2003). Nesse sentido, a maior abundância total da comunidade de pequenos mamíferos não-voadores nas matas secundárias na Reserva Florestal do Morro Grande está provavelmente associada ao aumento de produtividade e disponibilidade de recursos alimentares para espécies não dependentes de recursos restritos ou específicos. Este parece ser o caso dos marsupiais Marmosops incanus e Gracilinanus microtarsus, que ocupam o sub-bosque da floresta, e dos roedores Akodon montensis, Delomys sublineatus e Oryzomys angouya, que são bem mais freqüentes nas áreas secundárias da Reserva Florestal do Morro Grande.

A maior abundância em áreas de florestas mais jovens levou à idéia de que a comunidade de pequenos mamíferos não-voadores de florestas Neotropicais seria formada de espécies adaptadas a habitats secundários (Stallings 1988), ou com grande resiliência a distúrbios de habitat (Malcolm 1997b). Porém, como observado para outros grupos como borboletas (Hill et al. 1995) e aves (Aleixo 1999), os resultados encontrados na Reserva Florestal do Morro Grande indicam que existem espécies de pequenos mamíferos não-voadores da Mata Atlântica associadas a áreas de mata madura. Dentre as sete espécies que não foram encontradas em matas secundárias, estão espécies relativamente comuns e encontradas em todos os sítios de florestas maduras da Reserva Florestal do Morro Grande, como os marsupiais Monodelphis scalops e Philander frenata e o roedor Rhipidomys cf. mastacalis. As outras quatro espécies (Marmosops paulensis, Blarinomys breviceps, Oxymycterus dasytrichus e a espécie não descrita) foram raras também nas florestas maduras, tendo sido registradas em apenas um sítio a partir de um único indivíduo capturado. Além das espécies exclusivas, o roedor terrestre Thaptomys nigrita e o roedor arborícola Juliomys pictipes ocorreram tanto em áreas maduras quanto secundárias, mas com maior freqüência nas matas maduras. 
Dentre as espécies associadas às áreas maduras, três (T. nigrita, M. scalops e B. breviceps) apresentam características morfológicas que indicam o hábito terrestre associado ao folhiço (cauda curta, olhos e orelhas pequenos e garras fortes) e que poderiam depender do maior volume e umidade da serrapilheira das florestas maduras. Outras três espécies ( $R$. mastacalis, J. pictipes e a espécie não descrita) são roedores com adaptações morfológicas à vida arborícola (pés curtos e largos e cauda longa) que poderiam depender do maior número de árvores grandes ou da maior complexidade dos estratos superiores das florestas maduras.

As espécies mais comuns na Reserva Florestal do Morro Grande são o roedor terrestre Oryzomys russatus, o marsupial escansorial Marmosops incanus e o roedor terrestre Delomys sublineatus, todos representando mais de $10 \%$ do total de indivíduos capturados (Tabela 1). Oryzomys russatus é a espécie mais comum nos dois tipos de floresta, seguida de outro roedor (Thaptomys nigrita) nas matas maduras e do marsupial Marmosops incanus em áreas secundárias. De fato, uma espécie terrestre do gênero Oryzomys tende a ser uma das duas espécies mais comuns em áreas de Mata Atlântica madura e contínua, independentemente da altitude ou da região geográfica (Olmos 1991, Bergallo 1994, Bergallo \& Magnusson 1999, Pardini 2004). Em áreas de Mata Atlântica onde a vegetação foi fragmentada e/ ou alterada, as espécies do gênero Oryzomys perdem importância para outras espécies, sejam elas dos gêneros Akodon e Oligoryzomys e/ ou marsupiais que ocupam o sub-bosque (Fonseca \& Kierulff 1989, Stallings 1989, Paglia et al. 1995, Stevens \& Husband 1998, Pardini et al. no prelo). No Sul da Bahia, espécies dos gêneros Oryzomys, Thaptomys, Marmosops e Monodelphis são mais comuns em interiores de floresta madura, enquanto espécies de roedores dos gêneros Akodon e Oligoryzomys, e marsupiais dos gêneros Marmosa e Micoureus estão associadas a áreas alteradas, sejam elas bordas de fragmentos de mata, matas secundárias ou plantações sombreadas de cacau (Pardini 2004).

Talvez o que mais chame a atenção sobre a fauna de pequenos mamíferos não-voadores da Reserva Florestal do Morro Grande seja a riqueza da comunidade, que variou de 11 a 18 espécies por sítio de amostragem com média de 12,7 nas matas secundárias e de 15,7 nas matas maduras. A riqueza de pequenos mamíferos não-voadores em outros 40 sítios de Mata Atlântica já estudados, situados em áreas em diversos graus de preservação, variou de 3 a 16 com moda de 11 espécies por sítio (Vieira 1999). Em levantamento bibliográfico de inventários realizados em áreas maiores e mais bem preservadas de Mata Atlântica (Apêndice 1), a riqueza de pequenos mamíferos não-voadores apresenta faixa semelhante de variação, de 2 a 16 espécies. Assim, dois sítios de amostragem no Morro Grande abrigam o maior número local de espécies de pequenos mamíferos nãovoadores entre as áreas inventariadas na Mata Atlântica.
Além disso, o número total de espécies de pequenos mamíferos não-voadores na Reserva Florestal do Morro Grande (23) fica atrás apenas do observado para o Parque Estadual de Intervales (29 espécies, Vivo \& Gregorin 2001) e para o Parque Nacional do Itatiaia (33 espécies, Geise et al. 2004). No entanto, estes dois Parques tem área protegida muito maior, incluem diversidade de habitats e variação altitudinal muito mais amplas e seus inventários compreendem o acúmulo de dados de vários pesquisadores em diferentes épocas. Nos dois casos, a riqueza local de espécies é menor do que na Reserva Florestal do Morro Grande, variando de 11 a 16 em Intervales (Vieira \& Monteiro-Filho 2003) e de 3 a 7 no Parque Nacional de Itatiaia (Geise et al. 2004). Além disso, o número estimado de espécies para a Reserva Florestal do Morro Grande é equivalente ao registrado para estas duas áreas de Mata Atlântica $(29,4)$.

O maior esforço amostral e, em particular, a inclusão de armadilhas de queda na amostragem da Reserva Florestal do Morro Grande, pode ser uma das explicações para a alta riqueza de espécies em comparação a outros levantamentos, porém provavelmente não é a única. É o que indica o menor número de espécies (média por sítio de amostragem e riqueza observada e estimada considerando o total dos sítios amostrados) no levantamento realizado na Reserva Biológica de Una em área de baixada no Sul da Bahia, que incluiu armadilhas de queda e um esforço equivalente ao realizado na Reserva Florestal do Morro Grande (Pardini 2004). De fato, em trabalho realizado em vários dos maiores remanescentes da Bahia, Moura (2003) conclui que as áreas de Mata Atlântica do Nordeste abrigam comunidades de pequenos mamíferos não-voadores com composição diferente, mas em geral menos ricas, em relação às áreas mais ao sul da Mata Atlântica.

Além das diferenças associadas aos componentes biogeográficos do sul e norte (Costa 2003), padrões altitudinais de variação do número de espécies de pequenos mamíferos não-voadores já foram descritos para a Mata Atlântica (Bonvicino et al. 1997, Vieira 1999, Geise et al. 2004). Vieira (1999) comparou 40 sítios de regiões diferentes da Mata Atlântica e concluiu que florestas de baixada em geral abrigam um maior número de espécies. Porém, os sítios comparados diferiam também quanto ao grau de perturbação, estádios sucessionais e fragmentação. Os dados de 20 localidades (Apêndice 1) que abrangem diferentes regiões da Mata Atlântica, mas estão localizadas em grandes remanescentes de matas tardias ou maduras, indicam padrão inverso e estão de acordo com trabalhos recentes que têm demonstrado maior riqueza de pequenos mamíferos nãovoadores em altitudes intermediárias (500 a 1500 m), tanto na Mata Atlântica (Vieira \& Monteiro-Filho 2003, Geise et al. 2004), como em outras florestas tropicais (Goodman et al. 1999, Heaney 2001, Nor 2001, Sánchez-Cordero 2001, McCain 2004). Os 13 sítios localizados em áreas de baixada apresentam de 2 a 11 espécies de pequenos mamíferos não- 
voadores, enquanto os sete sítios localizados a altitudes maiores apresentam riqueza variando de 4 a 18 espécies (Apêndice 1). A riqueza de sítios localizados a altitudes maiores variando de 1000 a 2700 m na Serra do Caparaó não apresenta relação consistente com a altitude (Bonvicino et al.1997). Assim, a elevada riqueza de espécies de pequenos mamíferos não-voadores da Reserva Florestal do Morro Grande deve estar relacionada à inclusão de armadilhas de queda na amostragem, à localização mais ao sul na Mata Atlântica e à altitude intermediária.

\section{Distribuição espacial da diversidade de pequenos mamíferos não-voadores na Reserva Florestal do Morro Grande}

Apesar do maior número de espécies na Reserva Florestal do Morro Grande em relação à Reserva Biológica de Una, tanto localmente $(\alpha 1, \alpha 2, \gamma)$ como entre locais $(\beta 1, \beta 2)$, a distribuição da diversidade proporcionalmente à diversidade total é semelhante entre as duas áreas (Figura 2), o que indica que processos ecológicos semelhantes determinam a distribuição nas micro e meso escalas analisadas. Nos dois casos, a maior parte da diversidade total das reservas $(\gamma)$ é encontrada localmente nos sítios ( $\alpha 1$ ), e o turnover de espécies é maior entre sítios do mesmo habitat do que entre habitats. Estes resultados devem estar associados a dois fatores principais. Em primeiro lugar, à semelhança estrutural dos habitats analisados (florestas maduras e secundárias, e florestas de interior e de borda), já que tipos vegetacionais estruturalmente bem distintos abrigam comunidades de pequenos mamíferos não-voadores bastantes distintas na Mata Atlântica (Bonvicino et al. 1997, Vieira 1999, Umetsu 2005) ou no Cerrado (Alho 1981, Alho et al. 1986, Lacher et al. 2001). Em segundo lugar, estes resultados indicam a importância da heterogeneidade da floresta em micro escala, a qual deve levar a distribuições em manchas de espécies associadas a determinados microhabitats, e ao turnover relativamente grande de espécies entre sítios de um mesmo habitat. Esta indicação chama a atenção para a lacuna e a importância de dados relacionados à ecologia básica das espécies de pequenos mamíferos nãovoadores. Pouco conhecemos sobre o uso e a exigência de recursos das espécies de pequenos roedores e marsupiais da Mata Atlântica, ou da influência de fatores ambientais que variam em micro escala sobre a distribuição das espécies (Vieira 2003). Os resultados aqui apresentados indicam que este tipo de informação é fundamental para compreendermos os processos associados à distribuição da diversidade em áreas de Mata Atlântica.

\section{Distribuição temporal da diversidade e abundância de pequenos mamíferos não- voadores na Reserva Florestal do Morro Grande}

Grandes variações interanuais de abundância parecem ser comuns para as espécies de pequenos mamíferos não-voadores da Mata Atlântica e, em alguns casos, são maiores do que as variações sazonais, como indicam os poucos trabalhos de longo prazo realizados até o momento (Cerqueira et al. 1993, Gentile et al. 2000, revisão em Vieira 2003). A inexistência de variações interanuais significativas para a Reserva Florestal do Morro Grande pode estar associada à análise de dois períodos com apenas um ano de intervalo (Tabela 3). Por outro lado, pode também estar associada a uma maior estabilidade das populações e comunidades em áreas contínuas de Mata Atlântica em comparação a áreas mais perturbadas, já que os poucos estudos de longo prazo foram realizados em áreas com algum grau de perturbação e fragmentação (Cerqueira et al. 1993, Gentile et al. 2000). A abundância das espécies e a composição e a riqueza das comunidades de fragmentos pequenos e isolados próximos à Reserva Florestal do Morro Grande, amostrados da mesma forma e nas mesmas épocas, são muito mais variáveis temporalmente em comparação aos sítios da Reserva (R. Pardini, dados não publicados), indicando que a fragmentação ou alteração da floresta pode levar a um aumento da instabilidade temporal das populações e comunidades.

Por outro lado, os resultados para a Reserva Florestal do Morro Grande (Tabela 4) confirmam o padrão de variação sazonal das populações de pequenos mamíferos nãovoadores com um pico de abundância no final da estação chuvosa ou início da estação seca (Olmos 1991, Bergallo 1994, Bergallo \& Magnusson 1999, Quental et al. 2001, revisão em Vieira 2003). É importante salientar, no entanto, que na Reserva Florestal do Morro Grande este pico de abundância das espécies coincide com, e provavelmente causa, um pico na abundância e na riqueza da comunidade. O número médio de espécies por sítio de amostragem obtido no final da época chuvosa foi quase o dobro daquele obtido ao final da época de seca (Tabela 4). Apesar de não ser possível inferir sobre a generalidade deste padrão já que outros trabalhos não analisaram variações sazonais de riqueza, é de se esperar que acréscimos na abundância favoreçam a capturabilidade das espécies aumentando o número de espécies capturadas. Assim, a época de levantamento pode ser mais uma fonte de variação na comparação da diversidade entre inventários na Mata Atlântica.

\section{Distribuição da diversidade e abundância de pequenos mamíferos não-voadores entre os métodos de amostragem}

Os dados aqui apresentados indicam que as armadilhas de queda de 60 l capturam um maior número de indivíduos e de espécies de pequenos mamíferos nãovoadores do que armadilhas Sherman. No total da amostragem para a Reserva Florestal do Morro Grande, as primeiras permitiram a inclusão de todas as espécies 
capturadas com armadilhas Sherman, além de 10 espécies não capturadas nestas armadilhas. Entre essas 10 espécies, estão tanto espécies relativamente comuns (com cinco ou mais indivíduos capturados, como M. scalops, Monodelphis sp, $P$. nigrispinus e Brucepattersonius soricinus) quanto espécies local ou regionalmente raras (R. rufescens, $B$. breviceps e espécie não descrita) e espécies que ocupam micro-habitas particulares (O. dasytrichus e $N$. squamipes). Além destas, três espécies ( $M$. incanus, $D$. aurita e $O$. nigripes) foram significativamente mais capturadas, e duas (T. nigrita e J. pictipes) tenderam a ser mais capturadas, em armadilhas de queda.

Estas diferenças refletem variações no mecanismo de captura das duas armadilhas e não são resultado do protocolo de amostragem, já que tanto a área amostrada, como o número de armadilhas e o esforço empregado foram maiores para as armadilhas Sherman do que para as de queda (duas linhas de 165 m, 48 armadilhas e 1008 armadilhasnoite para cada sítio com Sherman, e uma linha de 100 m, 11 armadilhas e 352 armadilhas-noite para cada sítio com armadilhas de queda). Armadilhas de queda, apesar de não serem as usualmente utilizadas para inventários de pequenos mamíferos não-voadores, são seguramente menos seletivas do que aquelas tradicionalmente utilizadas. O mecanismo de captura destas últimas depende da atração dos animais pela isca ou pela possibilidade de abrigo, enquanto as armadilhas de queda capturam todos os indivíduos que passam por onde elas foram instaladas (número este aumentado pela cerca que direciona os animais para a área das armadilhas). Além disso, cada armadilha de queda pode capturar mais de um indivíduo por noite, provavelmente refletindo melhor a freqüência ou abundância das espécies do que as armadilhas do tipo gaiola, nas quais a captura de um animal impossibilita a captura de outros. Por outro lado, uma vez que tenha sido capturado em uma armadilha Sherman, um pequeno mamífero dificilmente consegue escapar. Já em armadilhas de queda, o escape depende da habilidade de salto ou do tamanho das espécies e do tamanho dos baldes utilizados.

Independente do tamanho do balde, armadilhas de queda capturam mais freqüentemente espécies semifossoriais ou terrestres associadas ao folhiço da floresta do que armadilhas Sherman (espécies dos gêneros Monodelphis e Scolomys na Amazônia com baldes de 201 Hice \& Schmidly 2002; espécies dos gêneros Monodelphis, Thaptomys e Blarinomys na Mata Atlântica com baldes de 35 l - Pardini 2004; e espécies dos gêneros Monodelphis, Thaptomys, Blarinomys e Brucepattersonius - este trabalho). Na Reserva Florestal do Morro Grande, foram utilizados baldes de $60 \mathrm{l}$, mais profundos do que os usualmente utilizados em outros trabalhos com pequenos mamíferos não-voadores (Lyra-Jorge \& Pivello 2001, Hice \& Schmidly 2002, Pardini 2004). Estes permitiram a captura, tão ou mais freqüente do que em armadilhas Sherman, de espécies de grande porte (Didelphis aurita, por exemplo) e espécies escansoriais ou arborícolas, as quais são raramente ou não são capturadas em baldes menores (Lyra-Jorge \& Pivello 2001, Hice \& Schmidly 2002, Pardini 2004). Em conclusão, o uso de armadilhas de queda de $60 \mathrm{l}$ rendeu um número grande de indivíduos e espécies relativamente ao esforço realizado e permitiu a representação de espécies de hábitos variados, de terrestres a arborícolas, incluindo tanto as espécies usualmente capturadas nos tipos de armadilhas mais tradicionalmente utilizadas, como espécies raramente capturadas.

\section{Conclusões}

A Reserva Florestal do Morro Grande apresenta uma fauna de pequenos mamíferos não-voadores que inclui uma série de espécies raras e espécies características de matas mais maduras, sendo as espécies mais abundantes pertencentes aos gêneros Oryzomys, Thaptomys, Delomys e Marmosops que em geral dominam outras áreas grandes e bem preservadas de Mata Atlântica. Uma das características marcantes da fauna de roedores e marsupiais da Reserva é a alta riqueza de espécies, tanto no total como por sítio de amostragem, em comparação a outros 20 levantamentos realizados em áreas pouco alteradas de Mata Atlântica. O número elevado de espécies por nós registrado está provavelmente relacionado ao uso de armadilhas de queda de 601 na amostragem, já que este método mostrou-se muito mais eficiente do que as armadilhas de contenção viva tradicionalmente utilizadas em inventários de pequenos mamíferos não-voadores, capturando um número significativamente mais alto de indivíduos e de espécies. Mas possivelmente também tem como causas a localização da Reserva, já que a comparação com outros inventários indica que áreas mais ao sul da Mata Atlântica ou aquelas localizadas a altitudes entre 500 e 1500 m tendem a apresentar comunidades mais ricas. De qualquer forma, nossos resultados indicam a importância da Reserva Florestal do Morro Grande para a conservação de marsupiais e pequenos roedores da Mata Atlântica e sugerem que a Reserva seja vista como uma área prioritária para ações de conservação no Planalto paulista.

Apesar deste maior número de espécies, a partição aditiva indica um padrão semelhante de distribuição espacial da diversidade entre a Reserva Florestal do Morro Grande e a Reserva Biológica de Una, com a maior parte da diversidade sendo encontrada localmente nos sítios de amostragem, secundariamente entre sítios do mesmo habitat e apenas uma menor parte entre habitats. Este resultado indica a importância da heterogeneidade da floresta em micro escala para a distribuição das espécies e da diversidade, fator cuja influência ainda conhecemos muito pouco. A abundância e a riqueza da Reserva não variaram em dois anos de amostragem apesar de variações temporais interanuais grandes terem sido observadas para fragmentos adjacentes 
à Reserva, indicando que áreas contínuas de mata podem apresentar populações e comunidades mais estáveis no tempo. Entretanto, a abundância de várias espécies e a abundância total e a riqueza da comunidade variaram sazonalmente de acordo com dados já existentes na literatura, com valores mais altos no final da estação chuvosa e valores mais baixos no final da estação seca.

\section{Agradecimentos}

Agradecemos a A. Percequillo e R. Rossi pelo auxílio na identificação das espécies de pequenos mamíferos; a S.M de Souza, R. Braga-Neto, M.Dixo, J.M.B. Gheller, R.G. Pimentel, D. Munari, T. Püttker, K. Kato, F.S. Cunha e F.M.P. Salvestrini pela indispensável ajuda no campo; a Rogério Rossi e a um assessor anônimo, pela revisão de uma versão prévia deste manuscrito; à SABESP, pelo alojamento durante o trabalho de campo e permissão para trabalho na Reserva Florestal do Morro Grande; e a FAPESP - Fundação de Amparo à Pesquisa do Estado de São Paulo (01/13309-2, 02/10845, 02/02125-0, 02/ 02126-7), WWF-Brasil (CSR 293-2003) e CNPq(690144/01-6) pelos financiamentos e bolsas de estudo. Este trabalho fez parte do projeto temático BIOTA/FAPESP “Conservação da Biodiversidade em Paisagens Fragmentadas no Planalto Atlântico de São Paulo” (99/05123-4).

\section{Referências bibliográficas}

ALEIXO, A. 1999. Effects of selective logging on a bird community in the Brazilian Atlantic Forest. Condor 101:537-548.

ALHO, C.J.R. 1981. Small mammal populations of Brazilian cerrado: the dependence of abundance and diversity on habitat complexity. Rev. Bras. Biol. 41:223-230.

ALHO, C.J.R., PEREIRA, L.A. \& PAULA, A.C. 1986. Patterns of habitat utilization by small mammal populations in cerrado biome of central Brazil. Mammalia 50:447-460.

BERGALLO, H.G. 1994. Ecology of small mammal community in an Atlantic Forest area in southeastern Brazil. Stud. Neotrop. Fauna E.29:197-217.

BERGALLO, H.G. \& MAGNUSSON, W.E. 1999. Effects of climate and food availability on four rodent species in southeastern Brazil. J. Mammal. 80:472-486.

BONVICINO, C.R., LANGGUTH, A., LINDBERGH, S.M. \& PAULA, A.C.1997. An elevational gradient study of small mammals at Caparaó National Park, South eastern Brazil. Mammalia 61:547-560.

BREWER, S.W. \& REJMANEK, M. 1999. Small rodents as significant dispersers of tree seeds in a Neotropical forest. J. Veg. Sci. 10:165-174.

CASTRO, E.B.V. \& FERNANDEZ, F.A.S., 2004. Determinants of differential extinction vulnerabilities of small mammals in Atlantic forest fragments in Brazil. Biol. Conserv.119:73-80.
CERQUEIRA, R., FERNANDEZ, F.A.S. \& QUINTELA, M.F.S. 1990. Mamíferos da restinga de Barra de Maricá, Rio de Janeiro. Pap. Avulsos Zool. 37:141-157.

CERQUEIRA, R., GENTILE, R., FERNANDEZ, F.A.S. \& D'ANDREA, P.S. 1993. A five-year study of an assemblage of small mammals in Southeastern Brazil. Mammalia 57:507-517.

COLGAN, W. \& CLARIDGE, A.W. 2002. Mycorrhizal effectiveness of Rhizopogon spores recovered from fecal pellets of small forest-dwelling mammals. Mycol. Res. 106:314-320.

COSTA, L. P. 2003. The historical bridge between the Amazon and the Atlantic Forest of Brazil: a study of molecular phylogeography with small mammals. J. Biogeogr. 30:71-86.

COSTA, L. P., LEITE, Y. L. R., FONSECA, G. A. B. \& FONSECA, M. T. 2000. Biogeography of South American forest mammals: endemism and diversity in the Atlantic Forest. Biotropica 32:872-881.

COLWELL, R.K. 2000. EstimateS: Statistical estimation of species richness and shared species from samples. Version 6.0b1. User's guide and application. (disponível em http://viceroy.eeb.uconn.edu/EstimateS; acesso em 15/12/2005).

CUNHA, A.A. \& VIEIRA, M.V. 2002. Support diameter, incline, and vertical movements of four didelphid marsupials in the Atlantic forest of Brazil. J. Zool. 258:419-426.

DE WALT, S.J., MALIAKAL, S.K. \& DENSLOW, J.S. 2003. Changes in vegetation structure and composition along a tropical forest chronosequence: implications for wildlife. Forest Ecol. Manag. 182:139-151.

FELICIANO, B.R., FERNANDEZ, F.A.S., FREITAS, D. \& FIGUEIREDO, M.S.L. 2002. Population dynamics of small rodents in a grassland between fragments of Atlantic Forest in southeastern Brazil. Mamm. Biol. 67:304-314.

FONSECA, G.A.B., HERRMANN, G., LEITE, Y.L.R., MITTERMEIER, R.A., RYLANDS, A.B. \& PATTON, J.L. 1996. Lista anotada dos mamíferos do Brasil. Occas. Pap. Conserv. Biol. 4:1-38.

FONSECA, G.A.B. \& KIERULFF, M.C.M. 1989. Biology and natural history of Brazilian Atlantic Forest small mammals. Bull. Fla. State Mus. Biol. Sci. 34:99-152.

GEISE, L, PEREIRA, L.G., BOSSI, D.E.\& BERGALLO, H.G. 2004. Patterns of elevational distribution and richness of nonvolant mammals in Itatiaia National Park and surroundings, in Southeastern Brazil. Braz. J. Biol. 64:1-15.

GENTILE, R., D’ANDREA, P.S., CERQUEIRA, R. \& MAROJA, L.S. 2000. Population dynamics and reproduction of marsupials and rodents in a Brazilian rural area: a five-year study. Stud Neotrop Fauna E. 35:1-9. 
GENTILE, R. \& FERNANDEZ, F.A.S.1999. Influence of habitat structure on a streamside small mammal community in a Brazilian rural area. Mammalia 63:29-40.

GOODMAN, S.M., CARLETON, M.D. \& PIDGEON, M. 1999. Rodents of the Reserve Naturelle Integrale d'Andohahela, Madagascar. Fieldiana, Zool. 94:217-249

GRELLE, C.E.V. 2002. Is higher taxon analysis an useful surrogate of species richness in studies of Neotropical mammal diversity? Biol. Conserv. 108:101-106.

GRELLE, C.E.V. 2003. Forest structure and vertical stratification of small mammals in a secondary Atlantic forest, southeastern Brazil. Stud Neotrop Fauna E. 38:81-85.

GRELLE, C.E.V. \& GARCIA, Q.S. 1999. Potential dispersal of Cecropia hololeuca by the common opossum (Didelphis aurita) in Atlantic forest, southeastern Brazil. Rev. Ecol.- Terre Vie 54:327-332.

GUARIGUATA, M.R. \& OSTERTAG, R. 2001. Neotropical secondary forest succession: changes in structural and functional characteristics. Forest Ecol. Manag. 148:185-206.

HEANEY, L R. 2001. Small mammal diversity along elevational gradients in the Philippines: an assessment of patterns and hypotheses. Global Ecol. Biogeogr. 10:15-39.

HICE, C.L. \& SCHMIDLY, D. 2002. The effectiveness of pitfall traps for sampling small mammals in the Amazon basin. Mastozool. Neotrop. 9:85-89.

HILL, J.K., HAMER, K.C., LACE, L.A. \& BANHAM, W.M.T. 1995. Effects of selective logging on tropical forest butterflies on Buru, Indonesia. J. Appl. Ecol. 32:754-760.

JANOS, D.P., SAHLEY, C.T. \& EMMONS, L.H. 1995. Rodent dispersal of vesicular-arbuscular mycorrhizal fungi in Amazonian Peru. Ecology 76:1852-1858.

LACHER, T.E. Jr. \& ALHO, C.J.R. 2001. Terrestrial small mammal richness and habitat associations in an Amazon Forest-Cerrado Contact Zone. Biotropica 33:171-181.

LANDE, R. 1996. Statistics and partitioning of species diversity, and similarity among multiple communities. Oikos 76:5-13.

LEITE, Y.L.R., COSTA, L.P. \& STALLINGS, J.R. 1997. Diet and vertical space use of three sympatric opossums in a Brazilian Atlantic forest reserve. J. Trop. Ecol. 12:435-440.

LYRA-JORGE, M.C. \& PIVELLO, V.R., 2001. Combining live trap and pitfall to survey terrestrial small mammals in savanna and forest habitats, in Brazil. Mammalia 65:524-530.

McCAIN, C.M. 2004. The mid-domain effect applied to elevational gradients: species richness of small mammals in Costa Rica. J. Biogeogr. 31:19-31.

MALCOLM, J.R. 1995. Forest structure and the abundance and diversity of Neotropical small mammals. In Forest canopies (M.D. Lowman \& N.M. Nadkarni, eds). Academic Press, San Diego, p.179-197.
MALCOLM, J.R. 1997a. Insect biomass in Amazonian forest fragments. In Canopy Arthropods (N.E. Stork, J. Adis \& R.K. Didham, eds). Chapman and Hall, London, p.510-533.

MALCOLM, J.R. 1997b. Biomass and diversity of small mammals in Amazonian forest fragments. In: Tropical forest remnants - ecology, management, and conservation of fragmented communities (W.F. Laurance \& R.O. Bierregaard, coords). The University of Chicago Press, Chicago, p.207-221.

MANGAN, S.A. \& ADLER, G.H. 2000. Consumption of arbuscular mycorrhizal fungi by terrestrial and arboreal small mammals in a Panamanian cloud forest. J. Mammal. 81:563-570.

METZGER, J.P., ALVES, L.A., CATHARINO, E.L.M., GOULART \& W., SIMÕES, S.J.C. 2006. Uma área de relevante interesse biológico, porém pouco conhecida: a Reserva Florestal do Morro Grande. Biota Neotrop. 6(2): http://www.biotaneotropica.org.br/ v6n2/pt/abstract?article+bn00406022006 (último acesso em 03/05/2006).

MOURA, R.T. 2003. Distribuição e ocorrência de mamíferos na Mata Atlântica do Sul da Bahia. In Corredor de biodiversidade da Mata Atlântica do sul da Bahia (P.I. Prado, E.C. Landau, R.T. Moura, L.P.S. Pinto, G.A.B. Fonseca, K.N. Alger, coords). IESB / CI / CABS / UFMG / UNICAMP, Ilhéus, publicação em CD-ROM.

NOR, S.M.D. 2001. Elevational diversity patterns of small mammals on Mont Kinabalu, Sabah, Malaysia. Global Ecol. Biogeogr. 10:41-62.

OLMOS, F. 1991. Observation on the behavior and population dynamics of some Brazilian Atlantic Forest rodents. Mammalia 55:555-565.

PAGLIA, A.P., MARCO, P., COSTA, F.M., PEREIRA, R.F. \& LESSA, G. 1995. Heterogeneidade estrutural e diversidade de pequenos mamíferos em um fragmento de mata secundária de Minas Gerais, Brasil. Rev. Bras. Zool. 12:67-79.

PASSAMANI, M., MENDES, S.L. \& CHIARELLO, A.G. 2000. Non-volant mammals of the Estação Biológica de Santa Lúcia and adjacent areas of Santa Teresa, Espírito Santo, Brazil. Bol. Mus. Biol. Mello Leitão 11/12:201-214.

PARDINI, R. 2004. Effects of forest fragmentation on small mammals in an Atlantic Forest landscape. Biodivers. Conserv. 13:2567-2586.

PARDINI, R., SOUZA, S.M., BRAGA-NETTO, R. \& METZGER, J.P. 2005. The role of forest structure, fragment size and corridors in maintaining small mammal abundance and diversity in a tropical forest landscape. Biol. Conserv. 124:253-266.

PARDINI, R., ROSSI, R.V. \& MUNARI, D.P. no prelo. Mamíferos não-voadores da Reserva Biológica do Alto da Serra de Paranapiacaba - uma comparação com outras localidades e com os registros do MZUSP. In Reserva Biológica do Alto da Serra de Paranapiacaba. (M.I.M.S. Lopes, M. Kirizawa \& M.M.R.F. Melo, coords). Secretaria do Meio Ambiente, São Paulo. 
PATTERSON, B.D. 2000. Patterns and trends in the discovery of new Neotropical mammals. Diversity Distrib. 6:145-151.

PERCEQUILLO, A.R., GONÇALVES, P.R. \& OLIVEIRA, J.A. 2004.The rediscovery of Rhagomys rufescens (Thomas, 1886), with a morphological redescription and comments on its systematic relationship based on morphological and molecular (cytochrome b) characters. Mamm. Biol. 69:238-257.

PEREIRA, L.G., TORRES, S.E.M., SILVA, H.S. \& GEISE, L. 2001. Non-volant mammals of Ilha Grande and adjacent areas in Southern Rio de Janeiro State, Brazil. Bol. Mus. Nac. 459:1-15.

PIMENTEL, D.S. \& TABARELLI, M. 2004. Seed dispersal of the palm Attalea oleifera in a remnant of the Brazilian Atlantic Forest. Biotropica 36:74-84.

PIRES, A.S., LIRA, P.K., FERNANDEZ, F.A.S., SCHITTINI, G.M. \& OLIVEIRA, L.C. 2002. Frequency of movements of small mammals among Atlantic Coastal Forest fragments in Brazil. Biol. Conserv. 108:229-237.

PIZO, M.A.1997. Seed dispersal and predation in two populations of Cabralea canjerana (Meliaceae) in the Atlantic Forest of southeastern Brazil. J. Trop. Ecol. 13:559-578.

QUADROS, J. \& CACERES, N.C. 2001. Ecologia e conservação de mamíferos na Reserva Volta Velha, SC, Brasil. Acta Biol. Leopoldensia 23:213-224.

QUADROS, J., CÁCERES, N.C., TIEPOLO, L.M. \& WÄNGLER, M.S. 2000. Mastofauna do Parque Estadual do Rio Guarani e área de influência da Usina Hidrelétrica de Salto Caxias, Baixo Rio Iguaçu, Estado do Paraná, Brazil. In Anais do II Congresso Brasileiro de Unidades de Conservação. (M.S. Milano \& V. Theulen, coords). Vol. II - trabalhos ténicos. Rede Nacional Pró-Unidades de Conservação / Fundação O Boticário de Proteção à Natureza, Campo Grande, p.822-829.

QUENTAL, T.B., FERNANDEZ, F.A.S. DIAS, A.T.C. \& ROCHA, F.S. 2001. Population dynamics of the marsupial Micoureus demerarae in small fragments of Atlantic Coastal Forest in Brazil. J. Trop. Ecol. 17:339-352.

SÁNCHEZ-CORDERO, V. \& MARTINEZ-GALLARDO, R. 1998. Postdispersal fruit and seed removal by forestdwelling rodents in a lowland rainforest in México. J. Trop. Ecol. 14:139-151.

SÁNCHEZ-CORDERO, V. 2001. Elevational gradients of diversity for rodents and bats in Oaxaca, Mexico. Global Ecol. Biogeogr. 10:63-76.

SILVA, C.R., PERCEQUILLO, A.R., IACK-XIMINES, G.E. \& VIVO, M. de. 2003. New distributional records of Blarinomys breviceps (Winge, 1888) (Sigmodontinae, Rodentia). Mammalia 67:147-152

SLADE, N.A. \& BLAIR, S.M., 2000. An empirical test of using counts of individuals as indices of population size. J. Mammal. 81:1035-1045.
STALLINGS, J.R. 1989. Small mammal inventories in an Eastern Brazilian Park. Bull. Fla. State Mus. Biol. Sci. 34:153-200.

STEVENS, S.M. \& HUSBAND, T.P. 1998. The influence of edge on small mammals: evidence from Brazilian Atlantic Forest fragments. Biol. Conserv. 85:1-8.

UMETSU, F. 2005. Pequenos mamíferos em um mosaico de habitats remanescentes e antropogênicos: qualidade da matriz e conectividade em uma paisagem fragmentada de Mata Atlântica. Dissertação de mestrado, Instituto de Biociências, Universidade de São Paulo, São Paulo.

VEECH, J.A., SUMMERVILLE, K.S., CRIST, T.O. \& GERING, J.C. 2002. The additive partitioning of species diversity: recent revival of an old idea. Oikos 99:3-9.

VIEIRA, E.M. 1999. Estudo comparativo de comunidades de pequenos mamíferos em duas áreas de Mata Atlântica situadas a diferentes altitudes no sudeste do Brasil. Tese de doutorado, Universidade Estadual de Campinas, Campinas.

VIEIRA, E.M. \& IZAR, P. 1999. Interactions between aroids and arboreal mammals in the Brazilian Atlantic rainforest. Plant Ecol. 145:75-82.

VIEIRA, E.M. \& MONTEIRO-FILHO, E.L.A. 2003. Vertical stratification of small mammals in the Atlantic rain forest of south-eastern Brazil. J. Trop. Ecol. 19:501-507.

VIEIRA, E.M., PIZO, M.A. \& IZAR, P. 2003a. Fruit and seed exploitation by small rodents of the Brazilian Atlantic forest. Mammalia 67:533-539.

VIEIRA, M.V. 2003. A dinâmica temporal e espacial de populações e comunidades animais da Floresta Pluvial Atlântica: pequenos mamíferos como um estudo de caso. In: Ecossistemas Brasileiros: Manejo e Conservação (V. Claudino-Sales, coord.). Expressão Gráfica e Editora, Fortaleza, p. 270-285.

VIEIRA, M.V., FARIA, D., FERNANDEZ, F., FERRARI, S., FREITAS, S., GASPAR, D.A., MOURA, R., OLIFIERS, N., OLIVEIRA, P.P. de, PARDINI, R., PIRES, A., RAVETTA, A., MELLO, M.A.R. de, RUIZ, C. \& SETZ, E. 2003 b. Mamíferos. In Fragmentação de Ecossistemas: Causas, efeitos sobre a biodiversidade e recomendações de políticas públicas. (D.M. Rambaldi \& D.A.S. Oliveira, coords). MMA/SBF, Brasília, p.125-151.

VIVO, M. 1997. A mastofauna da Floresta Atlântica: padrões biogeográficos e implicações conservacionistas. In Anais da $5^{\circ}$. Reunião Especial da SBPC - Floresta Atlântica: Diversidade Biológica e Sócio-Econômica. Blumenau, p.60-63.

VIVO, M. de \& GREGORIN, R. 2001. Mamíferos. In Intervales (C. Leonel, coord.). Fundação para a Conservação e a Produção Florestal do Estado de São Paulo, São Paulo, p.116-123.

http://www.biotaneotropica.org.br 
VOLTOLINI, J.C. 1997. Estratificação vertical de marsupiais e roedores na Floresta Atlântica do Sul do Brasil. Dissertação de mestrado, Universidade de São Paulo, São Paulo.

WHITTAKER, R.H., 1960. Vegetation of the Siskiyou Mountains, Oregon and California. Ecol. Monogr. 30:279-338.

Título: Pequenos mamíferos não-voadores da Reserva Florestal do Morro Grande - distribuição das espécies e da diversidade em uma área de Mata Atlântica

Autores: Pardini, R. \& Umetsu, F.

Biota Neotropica, Vol. 6 ( número 2 ): 2006

http://www.biotaneotropica.org.br/v6n2/pt/ abstract?article+bn00606022006

Recebido em 17/11/2004 - Versão reformulada recebida em 21/3/2005 - Publicado em 01/05/2006

ISSN 1676-0603

http://www.biotaneotropica.org.br 
Figura 1. Curva média de acúmulo do número de espécies de pequenos mamíferos não-voadores com o aumento de sítios de amostragem para duas áreas de Mata Atlântica com cerca de 10.000 ha. Círculo preto - Reserva Florestal do Morro Grande; círculo branco - Reserva Biológica de Una.

Figure 1. Mean accumulation curve of the number of non-volant small mammal species with increasing number of sampling sites for two areas of Atlantic Forest with around 10,000 ha. Full circle - Morro Grande Forest Reserve; empty circle - Una Biological Reserve.

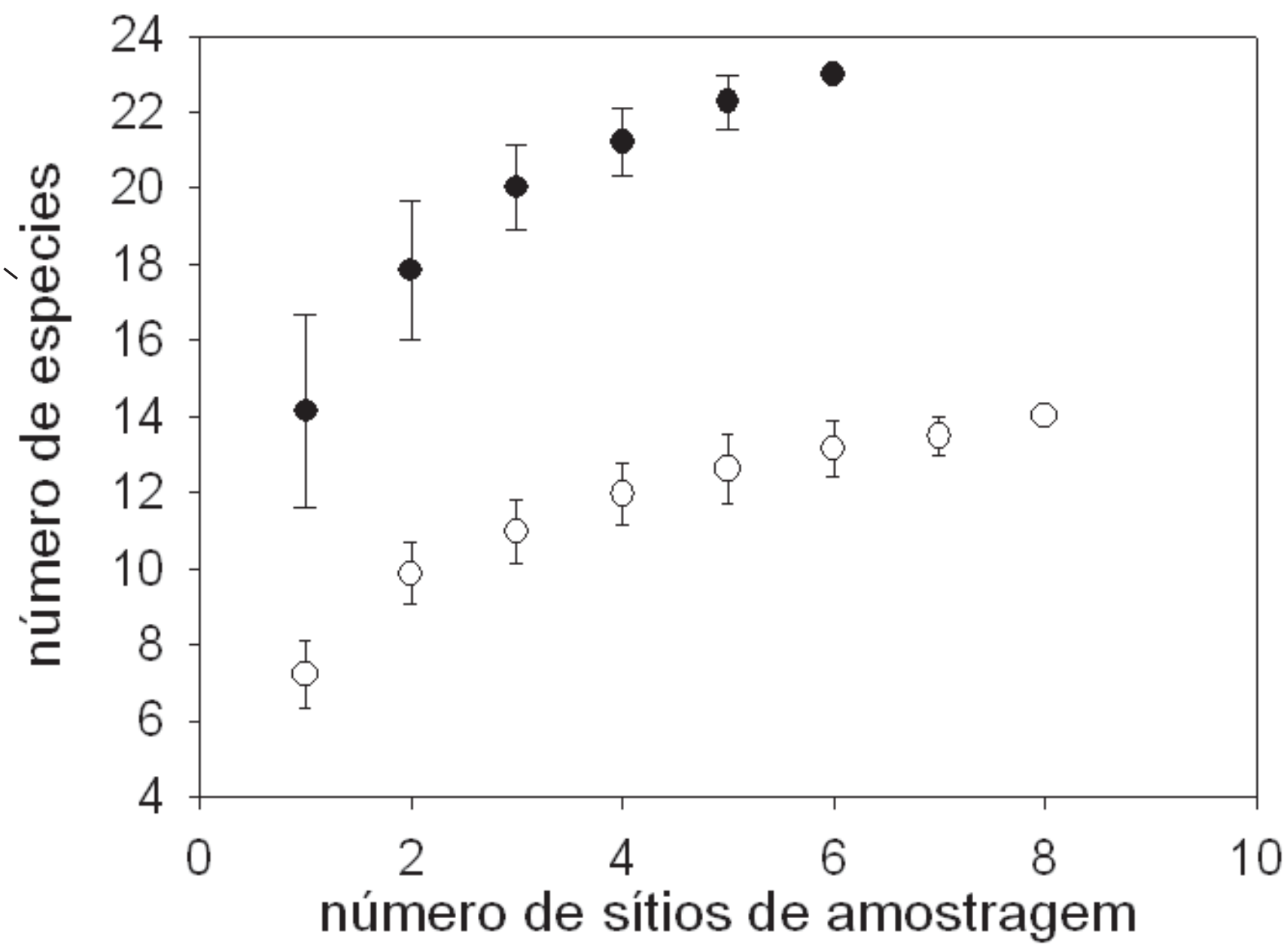

http://www.biotaneotropica.org.br 
Figura 2. Partição aditiva da diversidade total de espécies de pequenos mamíferos não-voadores em duas áreas de Mata Atlântica com cerca de 10.000 ha. $\gamma=$ número total de espécies registradas na reserva, $\alpha 1=$ média do número de espécies por sítio de amostragem, $\alpha 2=$ média do número de espécies por tipo de floresta (habitat), $\beta 1=\alpha 2-\alpha 1$ e $\beta 2=\gamma-\alpha 2$

Figure 2. Additive partitioning of the total diversity of non-volant small mammals in two areas of Atlantic Forest with around 10,000 ha. $\tilde{\boldsymbol{a}}=$ total number of species registered at the reserve, á1 = mean number of species in each sampling site, á $2=$ mean number of species in each type of forest (habitat), â1 = á2 - á1 e â2 = ̃̃ -á2.

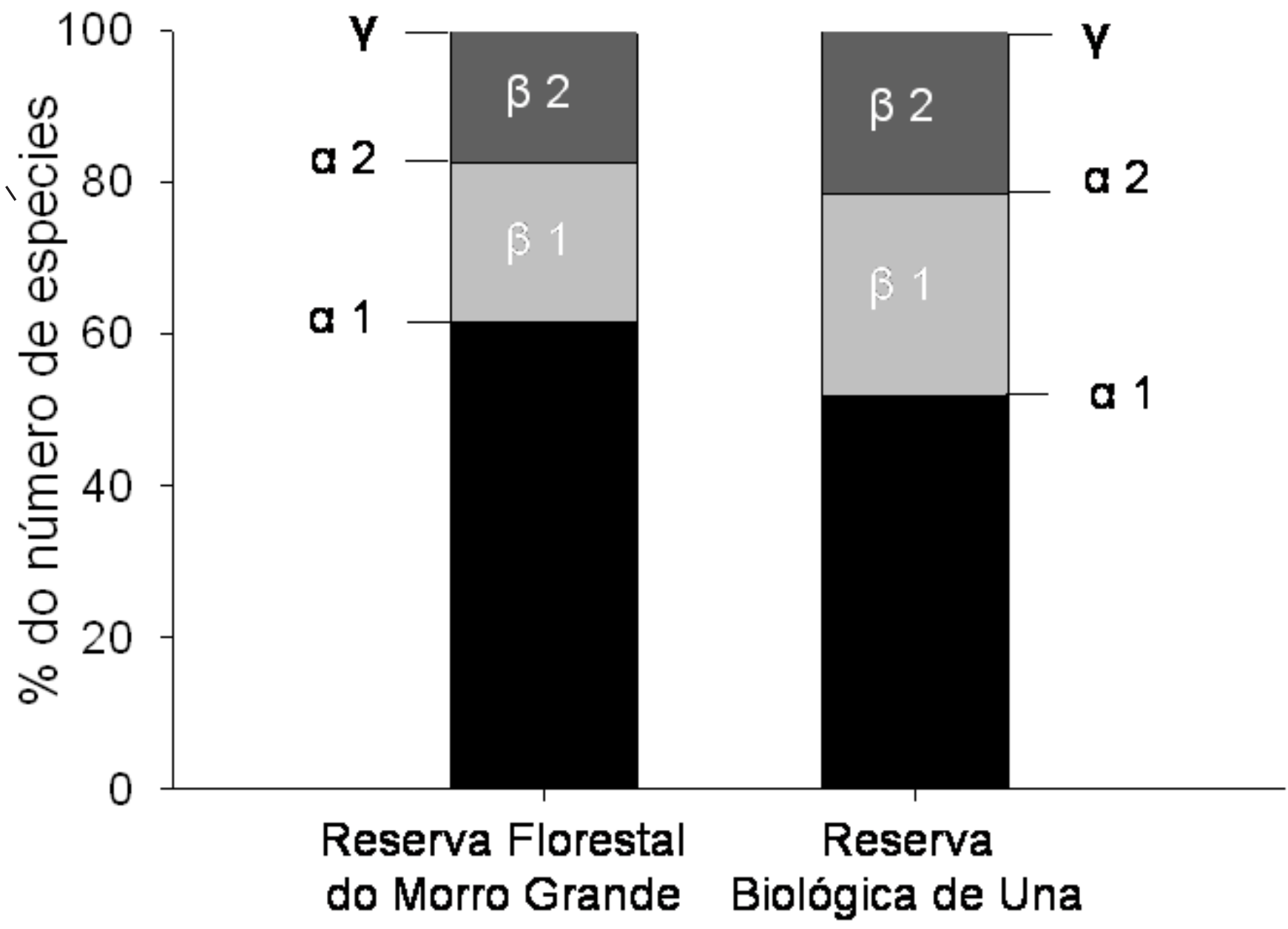

http://www.biotaneotropica.org.br 


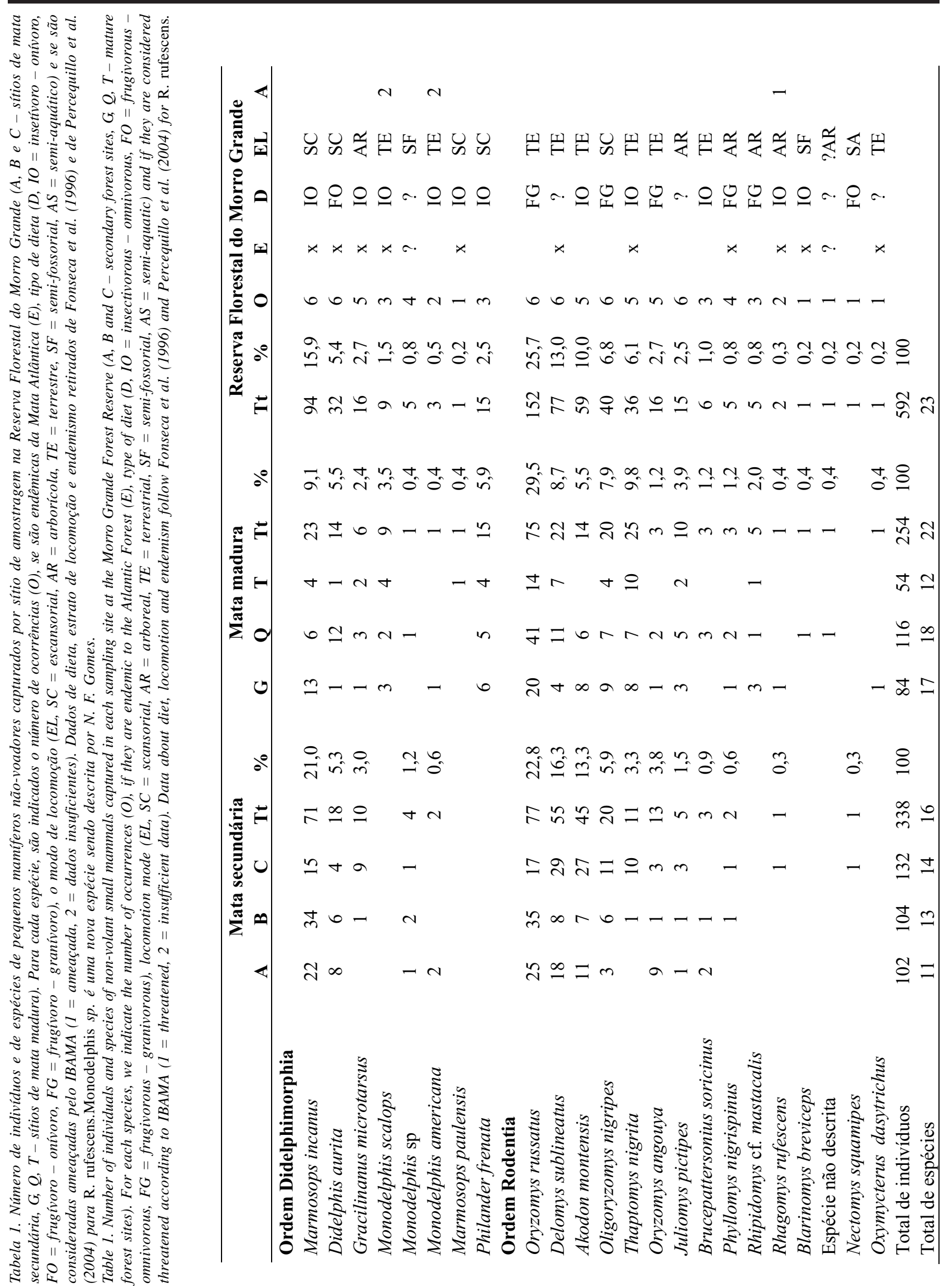


Tabela 2. Valores absolutos da partição aditiva da diversidade gama $(\gamma)$ de pequenos mamíferos não voadores para a Reserva Florestal do Morro Grande e para a Reserva Biológica de Una.

Table 2. Absolute values of the additive partitioning of gamma diversity ( $\gamma$ ) of non-volant small mammals for the Morro Grande Forest Reserve and for the Una Biological Reserve.

\begin{tabular}{lcc}
\hline & $\begin{array}{c}\text { Reserva Florestal do } \\
\text { Morro Grande }\end{array}$ & Reserva Biológica de Una \\
\hline diversidade alfa por sítio $(\alpha 1)$ & 14,17 & 7,25 \\
diversidade alfa por habitat $(\alpha 2)$ & 19,00 & 11,00 \\
diversidade beta entre sítios do mesmo habitat $(\beta 1)$ & 4,83 & 3,75 \\
diversidade beta entre habitats $(\beta 2)$ & 4,00 & 3,00 \\
diversidade gama $(\gamma)$ & 23,00 & 14,00 \\
\hline
\end{tabular}

http://www.biotaneotropica.org.br 
Tabela 3. Média (X), desvio-padrão (DP) e os resultados dos testes t pareados do número de indivíduos e de espécies de pequenos mamíferos não-voadores capturados com armadilhas de queda entre os dois anos de amostragem, considerando os seis sítios da Reserva Florestal do Morro Grande. * resultados significativos com $\alpha=0,05$. Monodelphis sp. é uma nova espécie sendo descrita por N. F. Gomes.

Table 3. Mean (X), standard-deviation (DP) and the results from paired t-tests of the number of individuals and species of non-volant small mammals captured with pitfall traps between the two years of sampling, considering the six sites of the Morro Grande Forest Reserve. * significant results considering $\alpha=0.05$.

\begin{tabular}{|c|c|c|c|c|c|c|}
\hline & \multicolumn{2}{|c|}{ Ano 1} & \multicolumn{2}{|c|}{ Ano 2} & \multirow[b]{2}{*}{$t$} & \multirow[b]{2}{*}{$p$} \\
\hline & $X$ & $D P$ & $X$ & $D P$ & & \\
\hline Akodon montensis & 1,83 & 1,47 & 4,00 & 4,24 & $-1,574$ & 0,176 \\
\hline Blarinomys breviceps & 0,00 & 0,00 & 0,17 & 0,41 & $-1,000$ & 0,363 \\
\hline Brucepattersonius soricinus & 0,17 & 0,41 & 0,83 & 1,17 & $-1,348$ & 0,235 \\
\hline Delomys sublineatus & 4,17 & 2,04 & 4,67 & 3,98 & $-0,374$ & 0,723 \\
\hline Didelphis aurita & 3,33 & 3,14 & 1,33 & 1,21 & 1,879 & 0,119 \\
\hline Espécie não descrita & 0,00 & 0,00 & 0,17 & 0,41 & $-1,000$ & 0,363 \\
\hline Gracilinanus microtarsus & 0,50 & 0,84 & 1,33 & 1,51 & $-1,052$ & 0,341 \\
\hline Juliomys pictipes & 1,17 & 1,60 & 1,00 & 0,63 & 0,222 & 0,833 \\
\hline Marmosops incanus & 7,83 & 7,94 & 4,83 & 2,79 & 0,910 & 0,405 \\
\hline Marmosops paulensis & 0,17 & 0,41 & 0,00 & 0,00 & 1,000 & 0,363 \\
\hline Monodelphis scalops & 1,50 & 1,76 & 0,00 & 0,00 & 2,087 & 0,091 \\
\hline Monodelphis americana & 0,33 & 0,52 & 0,00 & 0,00 & 1,581 & 0,175 \\
\hline Monodelphis sp. & 0,67 & 0,52 & 0,17 & 0,41 & 2,236 & 0,076 \\
\hline Nectomys squamipes & 0,00 & 0,00 & 0,17 & 0,41 & $-1,000$ & 0,363 \\
\hline Oligoryzomys nigripes & 3,50 & 1,52 & 2,50 & 1,05 & 1,581 & 0,175 \\
\hline Oryzomys angouya & 1,83 & 2,79 & 0,67 & 0,82 & 1,234 & 0,272 \\
\hline Oryzomys russatus & 5,33 & 3,72 & 3,33 & 0,82 & 1,491 & 0,196 \\
\hline Oxymycterus dasytrichus & 0,17 & 0,41 & 0,00 & 0,00 & 1,000 & 0,363 \\
\hline Philander frenata & 0,33 & 0,52 & 0,17 & 0,41 & 1,000 & 0,363 \\
\hline Phyllomys nigrispinus & 0,33 & 0,82 & 0,50 & 0,55 & $-0,349$ & 0,741 \\
\hline Rhagomys rufescens & 0,00 & 0,00 & 0,33 & 0,52 & $-1,581$ & 0,175 \\
\hline Rhipidomys mastacalis & 0,00 & 0,00 & 0,50 & 0,55 & $-2,236$ & 0,076 \\
\hline Thaptomys nigrita & 3,50 & 2,88 & 1,33 & 1,21 & 2,137 & 0,086 \\
\hline Abundância & 42,17 & 12,73 & 28,67 & 10,41 & 2,067 & 0,094 \\
\hline Riqueza & 10,17 & 1,83 & 10,83 & 2,23 & $-0,830$ & 0,444 \\
\hline
\end{tabular}

http://www.biotaneotropica.org.br 
Tabela 4. Média (X), desvio-padrão (DP) e os resultados das ANOVAs de medidas repetidas do número de indivíduos e de espécies de pequenos mamíferos não-voadores capturados com armadilhas Sherman entre as três sessões de amostragem, considerando os seis sítios da Reserva Florestal do Morro Grande. * resultados significativos com $\alpha=0,05$.

Table 4. Mean (X), stardard-deviation (DP) and the results from repeated-measures ANOVAs of the number of individuals and species of nonvolant small mammals captured with Sherman traps among the three sessions of sampling, considering the six sites at the Morro Grande Forest Reserve. * significant results considering $\alpha=0.05$.

\begin{tabular}{|c|c|c|c|c|c|c|c|c|}
\hline & Julho & & Outubro & & Abril & & & \\
\hline & $X$ & $D P$ & $X$ & $D P$ & $X$ & $D P$ & $F$ & $p$ \\
\hline Akodon montensis & 2,17 & 1,72 & 0,83 & 1,60 & 1,00 & 2,00 & 1,979 & 0,295 \\
\hline Delomys sublineatus & 0,83 & 2,04 & 0,50 & 1,22 & 2,67 & 2,58 & 4,000 & 0,111 \\
\hline Oryzomys russatus & 3,33 & 1,86 & 4,83 & 2,71 & 8,50 & 5,01 & 7,908 & 0,062 \\
\hline Thaptomys nigrita & 0,17 & 0,41 & 0,67 & 1,21 & 0,33 & 0,82 & 1,000 & 0,500 \\
\hline Marmosops incanus & 0,67 & 0,82 & 0,50 & 0,84 & 1,83 & 1,94 & 1,521 & 0,369 \\
\hline Philander frenata & 1,17 & 1,60 & 0,33 & 0,82 & 0,50 & 1,22 & 1,750 & 0,284 \\
\hline Abundância & 9,17 & 5,00 & 8,50 & 5,54 & 16,33 & 5,35 & 15,186 & $0,026^{*}$ \\
\hline Riqueza & 3,83 & 1,72 & 2,67 & 2,16 & 4,17 & 1,94 & 13,458 & 0,030 * \\
\hline
\end{tabular}


Tabela 5. Total, média (X), desvio-padrão (DP) e os resultados dos testes t pareados do número de indivíduos e de espécies de pequenos mamíferos não-voadores capturados nos seis sítios amostrados na Reserva Florestal do Morro Grande entre os dois tipos de armadilhas. * resultados significativos com $\alpha=0,05$. Monodelphis sp. é uma nova espécie sendo descrita por N. F. Gomes.

Table 5. Total, mean (X), stardard-deviation (DP) and the results from paired t-tests of the number of individuals and species of non-volant small mammals captured in the six sites sampled at the Morro Grande Forest Reserve between the two types of traps. * significant results considering $\alpha=0.05$.

\begin{tabular}{|c|c|c|c|c|c|c|c|c|}
\hline & \multicolumn{3}{|c|}{ Armadilhas de Queda } & \multicolumn{3}{|c|}{ Armadilhas Sherman } & \multirow[b]{2}{*}{$t$} & \multirow[b]{2}{*}{$p$} \\
\hline & Total & $X$ & $D P$ & Total & $X$ & $D P$ & & \\
\hline \multicolumn{9}{|l|}{ Ordem Didelphimorphia } \\
\hline Marmosops incanus & 76 & 12,67 & 8,73 & 18 & 3,00 & 2,68 & 3,559 & $0,016^{*}$ \\
\hline Didelphis aurita & 28 & 4,67 & 3,98 & 4 & 0,67 & 0,82 & 2,547 & 0,051 \\
\hline Gracilinanus microtarsus & 11 & 1,83 & 1,47 & 5 & 0,83 & 2,04 & 1,732 & 0,144 \\
\hline Monodelphis scalops & 9 & & & & & & & \\
\hline Monodelphis sp & 5 & & & & & & & \\
\hline Phyllomys nigrispinus & 5 & & & & & & & \\
\hline Philander frenata & 3 & 0,50 & 0,84 & 12 & 2,00 & 2,53 & $-1,464$ & 0,203 \\
\hline Monodelphis americana & 2 & 0,33 & 0,52 & 1 & 0,17 & 0,41 & 1,000 & 0,363 \\
\hline Marmosops paulensis & 1 & & & & & & & \\
\hline \multicolumn{9}{|l|}{ Ordem Rodentia } \\
\hline Delomys sublineatus & 53 & 8,83 & 5,42 & 24 & 4,00 & 5,59 & 1,980 & 0,105 \\
\hline Oryzomys russatus & 52 & 8,67 & 4,27 & 100 & 16,67 & 8,36 & $-2,465$ & 0,057 \\
\hline Oligoryzomys nigripes & 36 & 6,00 & 2,10 & 4 & 0,67 & 1,21 & 8,000 & $<0.001^{*}$ \\
\hline Akodon montensis & 35 & 5,83 & 5,38 & 24 & 4,00 & 4,43 & 1,228 & 0,274 \\
\hline Thaptomys nigrita & 29 & 4,83 & 3,66 & 7 & 1,17 & 1,94 & 2,345 & 0,066 \\
\hline Oryzomys angouya & 15 & 2,50 & 3,39 & 1 & 0,17 & 0,41 & 1,606 & 0,169 \\
\hline Juliomys pictipes & 13 & 2,17 & 1,60 & 2 & 0,33 & 0,82 & 2,200 & 0,079 \\
\hline Brucepattersonius soricinus & 6 & & & & & & & \\
\hline Rhipidomys cf. mastacalis & 3 & 0,50 & 0,55 & 2 & 0,33 & 0,82 & 0,542 & 0,611 \\
\hline Rhagomys rufecens & 2 & & & & & & & \\
\hline Blarinomys breviceps & 1 & & & & & & & \\
\hline Espécie não descrita & 1 & & & & & & & \\
\hline Nectomys squamipes & 1 & & & & & & & \\
\hline Oxymycterus dasytrichus & 1 & & & & & & & \\
\hline Abundância & 388 & 70,83 & 16,88 & 204 & 34,00 & 14,20 & 6,194 & $0,002 *$ \\
\hline Riqueza & 23 & 13,67 & 2,88 & 13 & 5,83 & 1,33 & 7,069 & $0,001 *$ \\
\hline
\end{tabular}

http://www.biotaneotropica.org.br 


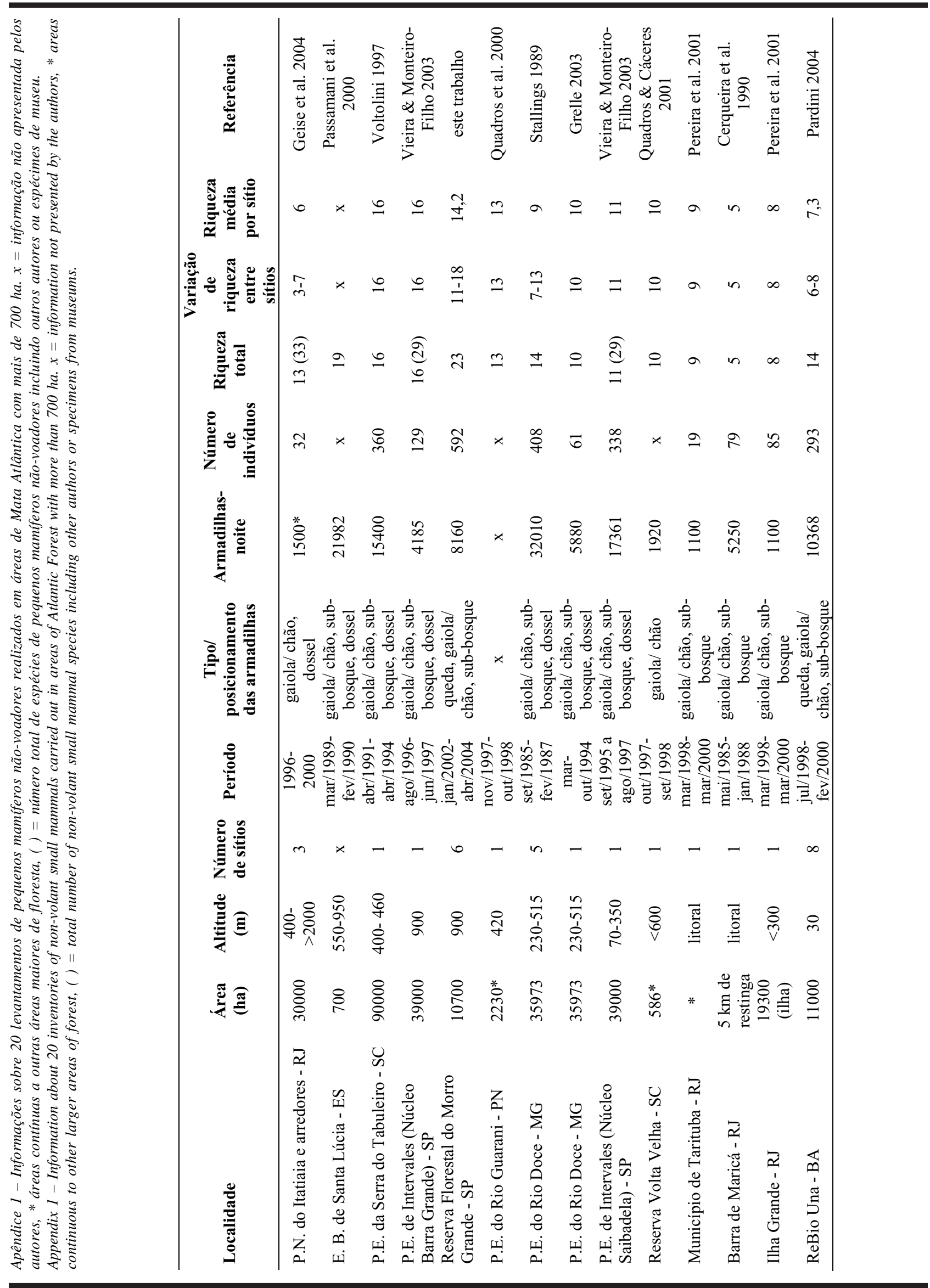

http://www.biotaneotropica.org.br 


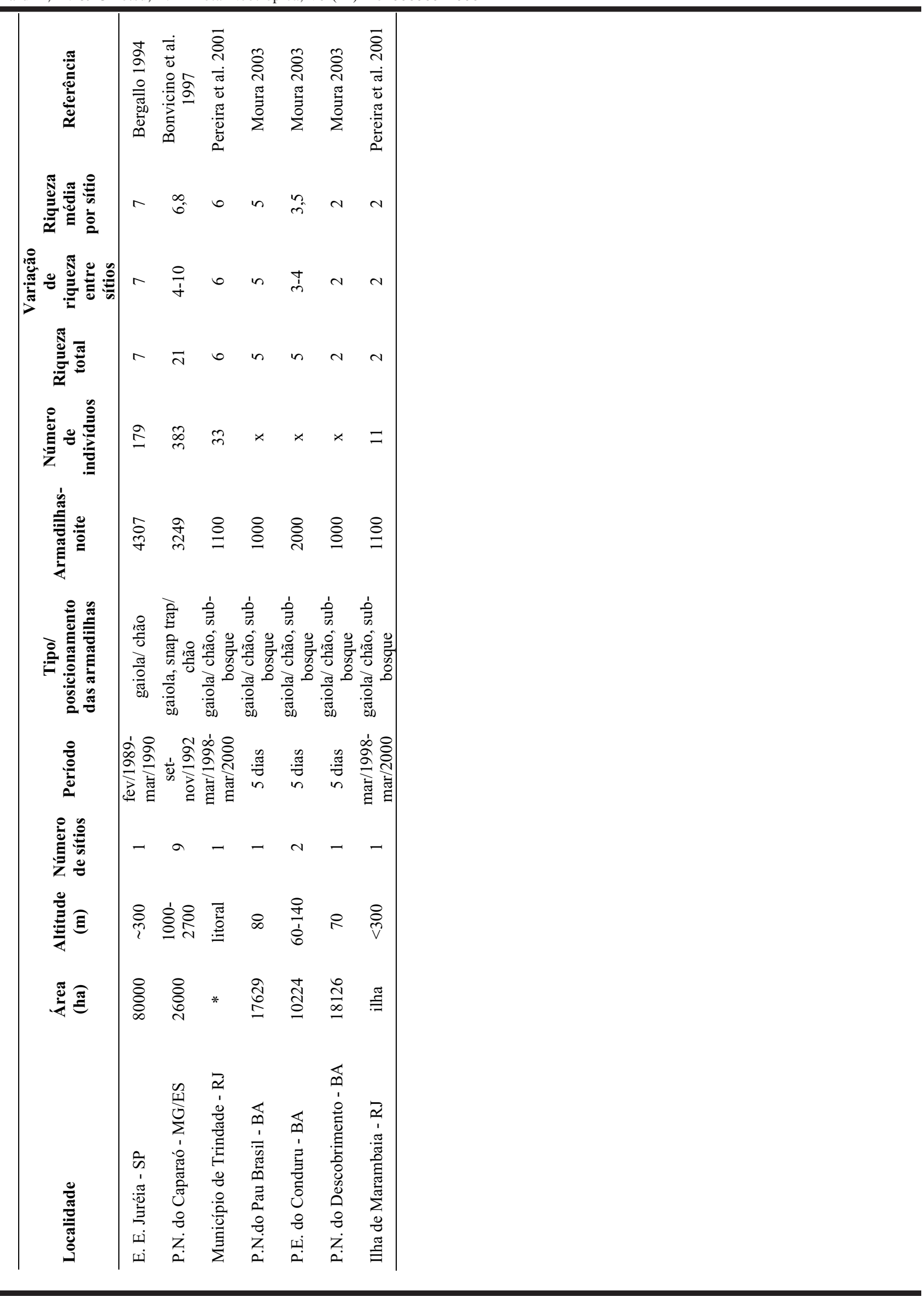

http://www.biotaneotropica.org.br 